\title{
An evaluation of the scanning electron microscope mirror effect to study viscoelastically prestressed polymeric matrix composites
}

\author{
Chao Ge ${ }^{\mathrm{a}, \mathrm{b}}$, Bing Wang ${ }^{\mathrm{a}}$, Kevin S. Fancey ${ }^{\mathrm{a}, \mathrm{c}, *}$ \\ ${ }^{a}$ School of Engineering \& Computer Science, University of Hull, HU6 7RX, UK \\ ${ }^{\mathrm{b}}$ School of Mechatronical Engineering, Beijing Institute of Technology, Beijing, 100081, \\ China \\ ${ }^{\mathrm{c}}$ GW Gray Centre for Advanced Materials, University of Hull, HU6 7RX, UK
}

\begin{abstract}
:
A viscoelastically prestressed polymeric matrix composite (VPPMC) is produced by applying tensile creep to polymeric fibres, the creep load being removed before the fibres are moulded into a resin matrix. Following matrix curing, the viscoelastically strained fibres impart compressive stresses to the surrounding matrix, counterbalanced by residual tension in the fibres. VPPMCs based on nylon 6,6 fibres in polyester resin have previously demonstrated improvements in mechanical properties of up to $50 \%$ compared with control (unstressed) counterparts. Although the associated viscoelastic recovery forces are understood, little is known of the fibre-matrix interactions relating to prestress within VPPMCs. This is addressed by investigating composite samples with the scanning electron microscope mirror effect (SEMME). By comparing results from VPPMC samples with their control counterparts, the findings suggest that there are $\sim 30 \%$ fewer trapped negative charges in the former, implying that the VPPMCs possess higher fibre-matrix interfacial strengths. Tensile test results on similar composite samples support these findings. The effects of resin porosity in SEMME data are also evaluated and our findings suggest that porosity can significantly increase charge trapping.
\end{abstract}

Keywords: Polymer-matrix composites (PMCs); scanning electron microscopy (SEM); interface; fibre-matrix bonding; residual stress; viscoelasticity.

(C) 2019. This manuscript version is made available under the CC-BY-NC-ND 4.0 license http://creativecommons.org/licenses/by-nc-nd/4.0/

\footnotetext{
* Corresponding author. Tel.: + 441482465071.

Email address: k.s.fancey@ hull.ac.uk (K.S. Fancey).
} 


\section{Introduction}

A viscoelastically prestressed polymeric matrix composite (VPPMC) is produced by subjecting polymeric fibres to tensile creep; the applied load is then removed before the fibres are moulded into a resin matrix. Following matrix curing, the viscoelastically strained fibres continue to attempt recovery through contraction. This imparts compressive stresses to the surrounding matrix, counterbalanced by residual tension in the fibres. VPPMCs based on nylon 6,6 fibres in polyester or epoxy resin have demonstrated improved mechanical properties, compared with control (unstressed) counterparts. Thus increases of up to $50 \%$ in impact toughness and flexural stiffness have been observed [1-6], while strength, modulus and energy absorption have exceeded $15 \%, 30 \%$ and $40 \%$ respectively from tensile tests [7].

A similar state of prestress can also be achieved with elastically prestressed PMCs (EPPMCs); here, the prestress is produced by maintaining an elastic tensile strain on fibres during matrix curing. For unidirectional continuous fibre EPPMCs, mechanical property improvements [8-12] are comparable to those of VPPMCs. There are however two potential drawbacks with EPPMCs. First, fibre length, orientation and spatial distribution are restricted by the need to apply fibre tension whilst the matrix cures; these restrictions can compromise fibre and mould geometries for more complex situations. Clearly, VPPMC processing has no such restrictions, as fibre stretching and moulding operations are decoupled; this enables total flexibility in product design, since there are no restrictions on fibre orientation and distribution or on component size and geometry. Second, since the matrix is polymeric, localised creep at the fibre-matrix interface regions can be expected, in response to compressive stresses imposed by the fibres. Therefore, the prestress effect within an EPPMC may deteriorate with time [2]. In contrast, there are long-term viscoelastic recovery mechanisms that occur within fibres such as nylon 6,6 after being subjected to appropriate creep conditions $[3,4,13]$. Here, accelerated ageing studies, based on time-temperature-superposition of nylon 6,6 fibres, have demonstrated no deterioration in VPPMC mechanical performance (Charpy impact toughness) over a duration equivalent to 25 years at a constant $50^{\circ} \mathrm{C}$ ambient [13]. In addition to mechanical property improvements and longevity, VPPMCs have been used to construct a bistable morphing structure [14,15]. Thus the design of VPPMC-based functional structures, such as morphing aerofoils, may be possible.

Although the main body of VPPMC research has been based on prestress provided by nylon 6,6 fibres [1-7,13], other researchers have successfully demonstrated VPPMCs based on bamboo, showing an increase in flexural toughness of $28 \%$ [16]. Most recently, increases of $20-40 \%$ in flexural modulus and impact toughness from VPPMCs based on ultra-high molecular weight polyethylene (UHMWPE) fibres have been achieved $[17,18]$. There have also been investigations into the force output-time characteristics from viscoelastically recovering fibres of nylon 6,6 [19] and UHMWPE $[17,18]$. For example, the viscoelastic recovery force from nylon 6,6 fibres was found to increase as a Weibull-based function with time $(t)$ [19] and, from recently published results in which the force output was monitored over a three year period, this has been predicted to reach a limiting value of $\sim 15 \mathrm{MPa}$ (contraction force relative to fibre cross- 
sectional area) as $t$ approaches infinity [20]. The initial growth in force from nylon 6,6 fibres is relatively high; thus, for example, after two weeks ( $336 \mathrm{~h}$ ), the output is $\sim 9$ $\mathrm{MPa}[19,20]$, i.e. $\sim 60 \%$ of the limiting value.

Despite the progress in research into the mechanical performance characteristics of VPPMCs, little is known of the role of the prestressing mechanism on fibre-matrix interactions within these composite materials. An investigative technique that may provide further insight is the scanning electron microscope mirror effect (SEMME), since this would enable the influence of prestress to be investigated from a nonmechanical perspective. Recently, an initial SEMME evaluation on VPPMC samples was performed [21] to determine the feasibility of this technique. In this paper, we aim to provide the groundwork for future directions of VPPMC study with the SEMME method, by focusing on essential requirements within the procedures, followed by new findings, from which the possible effects of electric charge interactions within these materials are discussed.

\section{Background}

The SEMME technique, developed during the 1990s [22,23], can provide information related to the trapping and mobility of electric charges within insulating materials. For fibre-reinforced composites, its use appears to have been limited to studies by Kechaou et al: they have investigated the influence of fibre-matrix interfaces on dielectric behaviour, to determine the mechanical effects of sizing on glass fibres moulded in epoxy resin [24-27] and drying of hemp fibres moulded in polypropylene [28]. In these studies, the fibre-matrix interface regions were observed to play a major role in the trapping or diffusion of charges, where charge diffusion is associated with high interface strength. Therefore, since viscoelastically generated stresses are created at the fibre-matrix interface regions in our composites, the SEMME technique may provide further insight into the role of these stresses on mechanical performance.

The principle of the SEMME method involves irradiating an insulating sample in an SEM with a high voltage (10s of $\mathrm{kV})$ over a controlled injection time, $t_{\mathrm{i}}$. During injection, negative charges are locally trapped and stabilised within the sample and these produce an electric field in the vacuum (sample) chamber of the SEM. If the sample is subsequently observed with a lower energy electron beam (100s of volts), electrons from this beam can be reflected from an equipotential surface produced by the electric field, as illustrated in Figure 1(a). Thus effectively, the arrangement is analogous to the behaviour of a convex mirror in visible light. The resulting mirror image can be observed on the SEM viewing screen as a distorted view of the SEM vacuum chamber, as shown in Figure 1(b).

The central black disc, shown in Figure 1(b), represents the exit aperture of the electron beam in the mirror image, and has an apparent diameter, $d$. The size of $d$ increases with decreasing electron beam energy, $V$, used for producing the mirror image, since the position of the equipotential surface causing beam reflection will 
depend on the beam energy used. Thus $1 / d=f(V)$ and the following relationship has been established by Vallayer et al [23]:

$$
\frac{1}{d}=\frac{4 L}{d_{\mathrm{r}}} \cdot \frac{2 \pi \varepsilon_{0}\left(\varepsilon_{\mathrm{r}}+1\right)}{K Q_{\mathrm{t}}} \cdot V
$$

Here, $d_{\mathrm{r}}$ is the real diameter of the exit aperture, $L$ the SEM working distance, $Q_{\mathrm{t}}$ the quantity of negative electric charge trapped and stabilised in the sample (which produces the electric field), and $K$ is a parameter dependent on SEM chamber characteristics; $\varepsilon_{0}$ and $\varepsilon_{\mathrm{r}}$ are the permittivities of free space and sample material (relative) respectively.

For mirror images produced at low beam voltages, a linear relationship can be expected from a plot of $1 / d$ versus $V$, and the quantity of trapped charge, $Q_{t}$, may be determined from the gradient. Thus a steeper gradient represents a lower value of $Q_{\mathrm{t}}$, implying that fewer charges are trapped and stabilised; instead, more charges diffuse through the material. For fibre-reinforced PMCs, there is evidence to suggest that diffusion of charges along the fibre-matrix interfaces, as opposed to trapping in these regions, corresponds to higher interfacial shear strength [24-28]. At higher beam voltages, the plot of $1 / d$ versus $V$ becomes non-linear: here, a sub-linear curve indicates a lateral spreading of trapped charges, whereas a super-linear curve implies a deeper dispersion of these charges [24].

Clearly, for VPPMC samples, the $1 / d$ versus $V$ characteristics, when compared with equivalent data from control samples, may provide information on the effects of prestress at the fibre-matrix interface regions. This in turn could lead to an improvement in our understanding of viscoelastically generated prestress mechanisms on composite mechanical properties.

\section{Experimental}

\subsection{Production of samples}

VPPMC sample preparation followed that described in previously published papers [1-7] and is briefly presented here. First, continuous multifilament nylon 6,6 yarn (140 filaments, $26 \mu \mathrm{m}$ fibre diameter, 94 tex), supplied by Ogden Fibres Ltd, UK, was annealed, to remove any previous stress history, in a fan assisted oven $\left(150^{\circ} \mathrm{C}, 0.5\right.$ h) for production of both test (prestressed) and control (no prestress) samples.

Following this, yarn for test samples was attached to a stretching rig and subjected to a $330 \mathrm{MPa}$ tensile creep stress for $24 \mathrm{~h}$ while yarn for control samples was positioned in close proximity to the stretching rig for exposure to the same ambient environment (20$\left.21^{\circ} \mathrm{C}, 30-40 \% \mathrm{RH}\right)$. Immediately after the load was removed, the test and control yarns were folded, cut into multiple (equal) lengths and brushed into flat ribbons (for fibre separation) ready for moulding. 
The matrix was a clear-casting resin, Reichhold Polylite 32032, mixed with 2\% MEKP catalyst, supplied by MB Fibreglass, UK. Gel time for this resin was $\sim 30 \mathrm{~min}$ and had sufficiently cured after 2 hours (at room temperature), to permit demoulding. Two identical aluminium moulds, each with a $10 \mathrm{~mm}$ wide and $3 \mathrm{~mm}$ deep channel, were used so that a strip of test and control material could be cast simultaneously from the same resin mix to minimise potential production-based variations. Following demoulding, the test and control strips were each cut into five samples with dimensions of $80 \times 10 \times 3.2 \mathrm{~mm}$. The batch (comprising five test and five control samples) was then held under a weighted steel strip for $24 \mathrm{~h}$ to prevent potential bending effects from internal stresses. The samples were stored in polythene bags (to minimise contamination from handling) at room temperature $\left(18-22^{\circ} \mathrm{C}\right)$ for 336 hours (2 weeks) prior to being used for tests. Although significant mechanical benefits (based on Charpy impact testing) have been observed from VPPMC samples only $12 \mathrm{~h}$ after moulding [1], a delay of 336 hours has been adopted in recent studies [5,29,30], as a 'standard' to ensure significant prestress levels, as outlined in Section 1. Two batches of samples, one with a fibre volume fraction $\left(V_{\mathrm{f}}\right)$ of $2 \%$ and the other with a $V_{\mathrm{f}}$ of $15 \%$ were produced; here, $V_{\mathrm{f}}$ was calculated as previously described [5]. These unidirectional continuous fibre composite samples were produced in accordance with our nylon 6,6 fibre-based samples used for Charpy impact testing, the lower $V_{\mathrm{f}}(2 \%)$ representing our 'standard' $V_{\mathrm{f}}$ for these purposes [1-5,13].

Although not reported during initial SEMME studies [21], the possibility of air bubbles within the resin, having some influence on charge trapping effects, was considered. Thus in the current study, a strip was cast to produce five $80 \times 10 \times 3.2 \mathrm{~mm}$ resin-only samples (i.e. no fibres), using the same moulding procedures outlined above. Then, following vigorous stirring of the same resin mix to induce bubbles, another strip was cast to produce a further five samples. To measure the volume fraction $\left(V_{\mathrm{b}}\right)$ of bubbles, a stereo microscope, at $15 \times$ magnification, was used to take photo images of the center resin-only sample from each of the two strips of five samples. For both samples, 10 photo images of $10 \times 8 \mathrm{~mm}$, were taken, to cover the whole sample surface. Processing, with ImageJ software, was then used to create sharpened, maximum contrast images of the bubbles, based on procedures previously published [31]. For each image, the number of bubbles and their dimensions were determined, to give 10 results of $V_{\mathrm{b}}$ from the sample; thus a mean value for $V_{\mathrm{b}}$ could then be calculated. It should be noted here that the photo images, representing a plane view, took no account of bubble depth from the sample surface. Thus some bubbles at greater depths may have been occluded by those closer to the surface and not observed. Although this is a potential source of error in $V_{\mathrm{b}}$, we suggest that the effect would have been minimal, as (i) the matrix resin gel time ( $\sim 30 \mathrm{~min})$ provided significant opportunities for bubble migration to the surface, and (ii) the high optical transparency of the resin facilitated observation to greater depths.

\subsection{SEMME tests}


To conduct the mirror effect tests, an S360 SEM (Electron Microscopy Ltd., Cambridge, UK) was employed. As a result of using open cast moulding, fibres tended to settle towards the bottom of the mould prior to curing, thereby increasing fibre spatial density towards one side of the samples. In this context, representative sample sections (test and control) have been previously published [5]. Thus all samples with fibres were tested with the fibre-rich surface (bottom of mould) facing uppermost to the electron beam. Resin-only samples were also tested in the same orientation.

Other SEMME investigations have utilised small parallelepiped samples, typically $\sim 10 \times 10 \times 1-5 \mathrm{~mm}$ thick [22-27,32]. High injection voltages $(30 \mathrm{kV})$ were used for a short duration, up to $200 \mathrm{~ms}$ [23-28], though longer injection times (10-20 s), at lower voltages $(<19 \mathrm{kV})$ have also been employed [32]. Thus for the relatively large beam-shaped samples used in our study, a number of preliminary trials were undertaken to determine the most appropriate procedures and settings. These trials were performed on a separate batch of samples $\left(2 \% V_{\mathrm{f}}\right)$, produced for this purpose.

Following the findings of the preliminary work, each sample (test, control or resin-only) was first irradiated in the SEM at $20 \mathrm{kV}$ in focused mode for 10 seconds $\left(t_{\mathrm{i}}\right)$ using a beam blanking device, the beam being targeted at the central region of the sample surface. The sample was then observed using nine lower energy voltage settings, ranging from $1000 \mathrm{~V}$ to the lowest attainable voltage of $200 \mathrm{~V}$, with the working distance, $L$, fixed at $23 \mathrm{~mm}$. The apparent diameter, $d$, was determined with the SEM cursor line measurement facility and a reading was performed within $30 \mathrm{~s}$ at each voltage setting; hence the highest voltage $(1000 \mathrm{~V})$ was applied first, as the resulting mirror image was most susceptible to being lost due to gradual charge depletion. The duration between irradiation and completing the nine readings was within five minutes. All samples were irradiated only once for these measurements, to avoid the risk of any influence caused by remaining charge effects from previous irradiation, including possible material degradation [28,32]. Thus for repeatability, at each voltage setting, a mean value of $d$ could be determined from five similar samples, to provide plots for test and control samples at $2 \%$ and $15 \% V_{\mathrm{f}}$ and the resin-only samples.

It should be noted here that for each sample to be irradiated, a 'dummy sample' was used for initial set-up. The dummy (identical to a $2 \% V_{\mathrm{f}}$ control sample) was permanently attached to one side of a $50 \mathrm{~mm}$ diameter rotatable sample holder within the SEM vacuum chamber, opposite to the sample under test. This arrangement enabled the beam to be accurately positioned on the dummy then, following beam blanking, the sample holder could be rotated through $180^{\circ}$ to perform the test.

\subsection{Tensile tests}

Referring to earlier mechanical performance tests, as summarised in Section 1, tensile testing can be expected to provide the most direct insight into fibre-matrix interactions from a mechanical perspective. This arises from the relatively simple state of stress to which tensile test samples are subjected. Although findings from previous tensile tests on VPPMCs [7] are informative, the sample dimensions, $V_{\mathrm{f}}$ values, 
moulding technique and matrix material were notably different to those of the current work. Therefore, two further batches of five test and five control composite samples (one batch at $2 \% V_{\mathrm{f}}$, the other at $15 \% V_{\mathrm{f}}$ ) were produced in accordance with Section 3.1. Although these two batches enable tensile test data to be acquired from samples identical to those used for the SEMME study, the optimum $V_{\mathrm{f}}$ for improved tensile properties was found to be $\sim 35 \%$ in Ref [7]; i.e. notably higher than the $V_{\mathrm{f}}$ values in the current work. Therefore, observed improvements in mechanical properties, especially at $2 \% V_{\mathrm{f}}$, may be small.

Tensile testing was performed with a Lloyd Instruments EZ-50 machine using a $50 \mathrm{kN}$ load cell. Each sample $(80 \times 10 \times 3.2 \mathrm{~mm})$ was clamped to provide a gauge length of $40 \mathrm{~mm}$ and a test speed of $10 \mathrm{~mm} / \mathrm{min}$ was adopted.

\section{Results and discussion}

\subsection{Sample appearance}

Figure 2(a) shows one of the composite sample batches. Both test and control samples appeared to be identical and, as demonstrated by the viewing angle of the figure, there was no sample distortion caused by prestress effects within the test samples. Although the matrix was transparent, fibres were not clearly observable within these samples. Similar nylon 6,6 fibre-based samples however, which have been photographed following Charpy impact tests, enable the fibres to be clearly seen within the regions of debonding, and these have been previously published $[2,4,5,13,20,29,30]$.

The resin-only samples are shown in Figure 2(b). For the low group, $V_{\mathrm{b}}$ was $0.008 \%$ with a range of $0.002-0.015 \%$ from the 10 image counts. Similarly, $V_{\mathrm{b}}$ for the high group was $0.933 \%$ with a range of $0.360-1.433 \%$. In the latter case, Figure 2(b) clearly shows (as expected) a narrow dispersion of the largest bubbles at the sample surfaces.

\subsection{SEMME image distortion}

Figure 3 shows representative mirror images of the SEM gun exit aperture. Figure 3(a) shows that aperture geometry was approximately circular (symmetrical) for resin-only samples, as were the $2 \% V_{\mathrm{f}}$ samples. For the $15 \% V_{\mathrm{f}}$ samples however, the aperture image was distorted to an elliptical shape as shown in Figure 3(b), possibly due to increased anisotropic charging effects from the higher $V_{\mathrm{f}}$ within the high geometrical aspect ratio of our samples. Since SEM cursor line measurement was confined to the horizontal axis, samples would have required an additional $90^{\circ}$ rotation for each voltage setting, which would have restricted the range of readings obtainable before the mirror image was lost. Therefore, readings for $d$ were restricted to the minor axis of the aperture image. Although smaller, more symmetrically shaped samples, as used by other investigators (reported in Section 3.2), may have enabled this to be avoided, the effects of fibre length on load transfer to the matrix must be considered. Fibre-matrix 
load transfer is generally characterised through the critical fibre length, which may exceed $25 \mathrm{~mm}$ for maximum mechanical performance for these VPPMCs [6]; thus use of smaller samples could be detrimental to prestress effects.

\subsection{Mirror curves}

All mirror curve data are summarised in Table 1. Figures 4(a) and (b) show the mirror curves from the test and control samples at $2 \%$ and $15 \% V_{\mathrm{f}}$. Both linear and curved regions are observed, which provide information on diffusion, stability and localisation of trapped charges within VPPMCs and their control counterparts. Although the origin of the linear regions is close to zero at $2 \% V_{\mathrm{f}}$ in Figure $4(\mathrm{a})$, there are negative intercepts on the $y$-axis for $15 \%$ in Figure 4(b) and this may be explained by the observed image distortion exemplified by Figure 3(b).

Despite distortion effects at $15 \% V_{\mathrm{f}}$, the gradient of the test samples in the linear region is notably steeper than that of the corresponding control samples at both $V_{\mathrm{f}}$ values. Since, at a particular $V_{\mathrm{f}}$, the test and control samples were moulded simultaneously and were tested under identical conditions, their permittivities are assumed to be similar. Thus according to Eq. (1), the steeper gradient for the test samples suggests that there are fewer trapped and stabilised charges, $Q_{\mathrm{t}}$, located in the injection point regions. Although there are a limited number of data points within the linear regions of Figure 4(a), approximate linear fits (with intercept fixed at zero) give gradients of 0.0066 (test) and 0.0049 (control). Thus based on Eq. (1), there is a decrease of $\sim 26 \%$ in the amount of trapped charges within the test samples, compared with corresponding control samples. Moreover, the gradients in Figure 4(b) give comparable values of 0.0061 (test) and 0.0042 (control), indicating a decrease in trapped charges of $\sim 31 \%$ for the test samples. As reported in Section 2, charge diffusion, which can be expected to occur along the fibre-matrix interfaces, as opposed to charge trapping, is associated with higher interfacial shear strength [24-28]. Therefore, these results suggest that viscoelastically generated prestress improves shear strength at the fibre-matrix interfaces. Charge diffusion along the fibre-matrix interfaces in favour of charge trapping must be attributed, directly or indirectly, to compressive stresses imparted by the viscoelastically strained fibres as they attempt strain recovery against the surrounding matrix material. It can be inferred that prestress effects reduce the availability of interfacial defects that could trap charges and this reduction in defects improves fibre-matrix interfacial adhesion. On a macroscopic level, an improvement in interfacial shear strength due to viscoelastically generated prestress can be readily associated with the observed increases in tensile strength and flexural stiffness from VPPMCs that have been previously reported [6,7]. More direct evidence is discussed in Section 4.4.

In addition to the linear regions, Figures 4(a) and (b) show that the curved regions are sub-linear for both test and control samples. As noted in Section 2, this implies a lateral spreading of trapped charges, which may be explained by the electrons being injected perpendicular to fibres; the charges will disperse along the fibre-matrix interfaces, to give a lateral spreading effect [24]. The transition from linearity in Figure 
4(b), at $\sim 800 \mathrm{~V}$, is higher than the $300-400 \mathrm{~V}$ in Figure $4(\mathrm{a})$ and this indicates that the size of the trapped charge zone is smaller [23] at the higher $V_{\mathrm{f}}(15 \%)$.

Figure 4(c) shows mirror plots for the resin-only samples with low and high bubble concentrations. Clearly, increasing $V_{\mathrm{b}}$ by two orders of magnitude enhances charge trapping; the gradients from Figure 4(c) are 0.0044 (low $V_{\mathrm{b}}$ ) and 0.0015 (high $V_{\mathrm{b}}$ ), which indicates almost a three-fold increase in trapped charges for the latter case. Interestingly, the transition from linearity for high $V_{\mathrm{b}}$, at $\sim 900 \mathrm{~V}$, is higher than the $\sim 400$ $\mathrm{V}$ for the low $V_{\mathrm{b}}$, thereby indicating that a high bubble content produces a smaller trapped charge zone. As reported in Section 3.1, all bubbles will have had a tendency to migrate towards the upper sample surface during casting. A possible explanation here is that bubbles in the high $V_{\mathrm{b}}$ case, being much larger, would therefore become less dispersed throughout the matrix; i.e. there would have been a much greater tendency for migration and confinement to the upper sample surface. Evidence of this can be seen in Figure 2(b), which also shows that dispersion of the larger bubbles is relatively narrow along the sample surface; hence both vertical and lateral confinement may have contributed to the smaller trapped charge zone.

Since the presence of bubbles can have a significant effect on charge trapping, the plots in Figures 4(a) and (b) require further consideration. Clearly, it can be expected that the addition of fibres with the resin during moulding will increase the probability of trapped air, so that a higher bubble content can be expected at $15 \% V_{\mathrm{f}}$. From Figure 4(b), the $15 \% V_{\text {f }}$ test samples show a decrease in trapped charges of $\sim 31 \%$, but this is little more than the reduction ( $26 \%$ ) observed at $2 \% V_{\mathrm{f}}$ in Figure $4(\mathrm{a})$; the test and control gradients in Figures 4(a) and (b) are also comparable. In contrast, at $15 \% V_{\mathrm{f}}$, more significant charge diffusion along the increased proportion of fibre-matrix interfaces might be expected, to give steeper test and control gradients and a greater decrease in trapped charges from the prestress effects. We suggest that the apparent disparity can be attributed to a higher bubble content at $15 \% V_{\mathrm{f}}$, as this could have reduced the gradient values in Figure 4(b) due to increased charge trapping. Also, the higher voltage for transition from linearity in Figure 4(b) suggests a smaller trapped charge zone, which concurs with the observation in Figure 4(c). A greater proportion of bubbles at $15 \% V_{\mathrm{f}}$ can be expected to be confined to the locality of the fibres, thereby creating a smaller trapped charge zone.

\subsection{Tensile test results}

Tensile test data from the $2 \%$ and $15 \% V_{\mathrm{f}}$ batches are presented in Table 2 and typical stress-strain plots are shown in Figure 5. In all cases, Figure 5 shows matrix fracture, followed by fibre strain and pull-out, then ultimately fibre fracture.

Of notable interest here is the region where matrix fracture occurs in Figures 5(a) and 5(b), as the following observations can be made. First, at both $V_{\mathrm{f}}$ values, the test samples fail at a higher stress than their control counterparts, as represented by the mean peak stress values in Table 2 increasing by $2.8 \%$ and $9.6 \%$ respectively for the $2 \%$ and $15 \% V_{\mathrm{f}}$ samples. Second, the corresponding strains are also higher, at $11.1 \%$ and $15.1 \%$ in Table 2 . Clearly, the increase in peak stress indicates that fibre-matrix 
adhesion is greater in the test samples; moreover, a higher strain level prior to matrix failure also suggests improved adhesion, since this must relate to the shear strain that is sustainable prior to the initiation of debonding at the fibre-matrix interfaces. Therefore, the observed improvement in fibre-matrix adhesion, as a result of viscoelastically generated prestress, must concur with the increased interfacial shear strength as discussed in Section 4.3.

Figures 5(a) and 5(b) show major differences in stress-strain characteristics following matrix fracture. In Figure 5(a), progressive fibre fracture occurs during fibre pull-out. Here, there are insufficient fibres at $2 \% V_{\mathrm{f}}$ to support the tensile load after matrix failure. Moreover, relatively few fibres may exacerbate the effects of localised variations in fibre strains caused, for example, by fibres not being perfectly parallel and aligned with the tensile load. In Figure 5(b), there are comparatively lower stress levels on individual fibres at $15 \% V_{\mathrm{f}}$, so they can more readily support the tensile load after matrix failure. This provides greater opportunity during pull-out for individual fibre strains to become equalised, so that the fibres fracture more collectively. The suggested increase in trapped air or bubbles between fibres at $15 \% V_{\mathrm{f}}$ (Section 4.3) could reduce fibre-matrix contact in some regions, which may also be expected to facilitate pull-out and equalisation of fibre strains, following matrix failure.

Progressive fibre fracture during pull-out at $2 \% V_{\mathrm{f}}$ gives a poorly defined final strain-to-failure (STF) in Figure 5(a). At $15 \% V_{\mathrm{f}}$ however, the test sample in Figure 5(b) shows a lower STF compared with the control and this is consistent with findings from previous work [7]. In Ref [7], analysis of mechanical tensile properties and surface characteristics of individual nylon 6,6 fibres revealed no significant changes (e.g. from work-hardening or surface damage) that could be attributed to the fibre stretching process; thus it was concluded that the observed reduction in STF from VPPMC samples could be attributed to prestress reducing any variations in deformation between fibres. In contrast with Ref [7] VPPMC results however, Figure 5(b) clearly shows the STF occurring beyond matrix fracture; though it is possible that the lower STF from the test sample may arise from matrix fragments, with improved fibre-matrix adhesion, impeding the strain in fibres prior to their fracture.

\subsection{Fibre-matrix adhesion}

Results from this study suggest that viscoelastically generated prestress improves shear strength at the fibre-matrix interfaces, possibly by a reduction in defects located at these regions, thereby increasing fibre-matrix interfacial adhesion. As highlighted in Section 1, VPPMC mechanical property improvements of up to 50\% have been obtained from previous studies. To date, these improvements have been attributed directly to physical prestress, with the following proposed mechanisms: (i) matrix compression impedes the influence of external loads, (ii) matrix compression reduces the extent of fibre fracture by attenuating dynamic overstress effects, (iii) residual fibre tension causes fibres to respond more collectively to external loads and (iv) fibre-matrix shear stresses trigger energy-absorbing fibre debonding instead of 
transverse fracture during impact events [4-7]. A prestress-induced enhancement of fibre-matrix adhesion may be considered to support these previously proposed effects.

The exact mechanism for enhanced bonding between the prestressing fibres and matrix is not clear. The mechanism may be purely mechanical; i.e. either it is (i) directly attributed to compressive stresses imparted by the viscoelastically strained fibres as they attempt strain recovery against the surrounding matrix material or (ii) the fibre surfaces are modified during the stretching process and that these changes may contribute to the observed effects. Nevertheless, we suggest that these are unlikely causes. As highlighted in Section 4.4, the tensile testing of individual nylon 6,6 fibres [7] and SEM analysis of fibre surfaces [7,30], revealed that these fibres are very smooth with few topographical features and no significant differences were observed between test and control fibres. The mechanism could instead have some indirect dependence on prestress; e.g. the compressive stresses influence the development of an interphase region, which in turn improves fibre-matrix shear strength. Although the fibres were not subjected to any surface treatments in this work, it is still possible for differential properties (an interphase) to occur at fibre-matrix interface regions [33].

SEMME studies suggest that electric charges play a significant role in the presence of defects. Therefore, the possibility that stretching and resulting viscoelastic recovery within the fibres might influence the presence of electric charges at the fibre surfaces, requires consideration. Mechanical stresses, when applied to an insulating material, lead to an injection of electric charges [26]. For intermediately disordered materials, such as polymers, low mobility leads to a strong localisation (trapping) of these charges [34], which enables the storage of significant polarisation energy ( $\sim \mathrm{eV}$ or more per trapped charge) [35]. It is therefore probable that charge trapping occurs when nylon fibres are subjected to creep, as required for VPPMC production. A subsequent external stress can result in the detrapping of charges and, if this is a sudden event, the release of stored polarisation energy can be detrimental to mechanical properties [26]. Thus following moulding, it is possible that viscoelastic recovery mechanisms within the fibres release the trapped charges (and polarisation energy) at the fibre-matrix interface regions and that interactions with these charges may lead to the influence of defects in the interface regions being reduced. We suggest however that viscoelastic activity during recovery would cause the trapped charges to be released gradually, as opposed to a sudden event. Although this is speculative, it is known that viscoelastic recovery within these fibres is a long-term phenomenon, which, even at an ambient temperature of $50^{\circ} \mathrm{C}$, exceeds 20 years [13]. From a mechanical perspective, viscoelastic activity is suggested to occur through the time-dependent triggering of molecular jumps, with longer term activity being represented by sites triggered through very long time constants [36]. In comparison, the dielectric and viscoelastic properties of a polymer reflect (differently) the same movement of molecular chains [37]; hence the release of trapped charges may concur with the triggering of molecular jumps during viscoelastic activity. Moreover, since permanent or quasi-permanent charge trapping might require a strong distortion of the lattice around each trapping site [34], this effect could be associated with longer term viscoelastic recovery. 


\section{Conclusions}

Although the SEMME technique seems only to have received limited attention within the field of composites research, our work provides additional evidence that the method can produce useful information on fibre-matrix interactions. This has led to a further understanding of the mechanisms that enable viscoelastically generated prestress to improve mechanical properties; a prestressing technique that also provides opportunities for total flexibility in product design. Specifically, our investigation has found evidence of there being fewer trapped negative charges in viscoelastically prestressed samples, implying that VPPMCs possess higher fibre-matrix interfacial strengths than their control (unstressed) counterparts. This is supported by tensile test results. Although the underlying causes are unknown, it may be speculated that viscoelastic activity within the fibres could influence electric charge interactions in the fibre-matrix interface regions. Moreover, our findings demonstrate that porosity within the matrix can significantly increase charge trapping.

\section{Acknowledgements}

Two of the authors (C.G., B.W.) would like to thank the China Scholarship Council for financial support. School of Engineering \& Computer Science support ( $\mathrm{PhD}$ degree fee waiver for B.W.) is also gratefully acknowledged. Special thanks go to Garry Robinson for technical advice and support.

\section{References}

[1] K.S. Fancey, Prestressed polymeric composites produced by viscoelastically strained nylon 6,6 fibre reinforcement, J. Reinf. Plast. Compos. 19 (2000) 12511266.

[2] K.S. Fancey, Fibre-reinforced polymeric composites with viscoelastically induced prestress, J. Adv. Mater. 37 (2005) 21-29.

[3] J.W.C. Pang, K.S. Fancey, An investigation into the long-term viscoelastic recovery of Nylon 6,6 fibres through accelerated ageing, Mat. Sci. Eng. A 431 (2006) 100-105.

[4] K.S. Fancey, Viscoelastically prestressed polymeric matrix composites potential for useful life and impact protection, Compos. Part B 41 (2010) 454 461.

[5] A Fazal, K.S. Fancey, Viscoelastically prestressed polymeric matrix composites -effects of test span and fibre volume fraction on Charpy impact characteristics, Compos. Part B 44 (2013) 472-479. 
[6] J.W.C. Pang, K.S. Fancey, The flexural stiffness characteristics of viscoelastically prestressed polymeric matrix composites, Compos. Part A 40 (2009) 784-790.

[7] J.W.C. Pang, K.S. Fancey, Analysis of the tensile behaviour of viscoelastically prestressed polymeric matrix composites, Compos. Sci. Technol. 68 (2008) 1903-1910.

[8] A.S. Hadi, J.N. Ashton, On the influence of pre-stress on the mechanical properties of a unidirectional GRE composite, Compos. Struct. 40 (1997) 305311.

[9] S. Motahhari, J. Cameron, Impact strength of fibre pre-stressed composites, J. Reinf. Plast. Comp. 17 (1998) 123-130.

[10] S. Motahhari, J. Cameron, Fibre prestressed composites: improvement of flexural properties through fibre prestressing, J. Reinf. Plast. Comp. 18 (1999) 279-288.

[11] L. H. Schlichting, M.A.C. de Andrada, L.C.C. Vieira, G.M.D. Barra, P. Magne, Composite resin reinforced with pre-tensioned glass fibers. Influence of prestressing on flexural properties, Dent. Mater. 26 (2010) 118-125.

[12] Y. Nishi, T. Okada, S. Okada, M. Hirano, M. Matsuda, A. Matsuo, M.C. Faudree, Effects of tensile prestress level on impact value of $50 \mathrm{vol} \%$ continuous unidirectional 0 degree oriented carbon fiber reinforced epoxy polymer (CFRP), Mater. Trans. 55 (2014) 318-322.

[13] K.S. Fancey, A. Fazal, Prestressed polymeric matrix composites: Longevity aspects, Polym. Compos. 37 (2016) 2092-2097.

[14] B. Wang, K.S. Fancey, A bistable morphing composite using viscoelastically generated prestress, Mater. Lett. 158 (2015) 108-110.

[15] B. Wang, C. Ge, K.S. Fancey, Snap-through behaviour of a bistable structure based on viscoelastically generated prestress, Compos. Part B 114 (2017) 23-33.

[16] H. Cui, M. Guan, Y. Zhu, Z. Zhang, The flexural characteristics of prestressed bamboo slivers reinforced parallel strand lumber (PSL), Key Eng. Mat. 517 (2012) 96-100.

[17] A. Fazal, K.S. Fancey, Viscoelastically generated prestress from ultra-high molecular weight polyethylene fibres, J. Mater. Sci. 48 (2013) 5559-5570.

[18] A. Fazal, K.S. Fancey, UHMWPE fibre-based composites: Prestress-induced enhancement of impact properties, Compos. Part B 66 (2014) 1-6.

[19] J.W.C. Pang, B.M. Lamin, K.S. Fancey, Force measurement from viscoelastically recovering Nylon 6,6 fibres, Mater. Lett. 62 (2008) 1693-1696.

[20] K.S. Fancey, Viscoelastically prestressed polymeric matrix composites: An overview, J. Reinf. Plast. Comp. 35 (2016) 1290-1301.

[21] C. Ge, B. Wang, K.S. Fancey, Techniques to investigate viscoelastically generated prestress in polymeric composites. In proceedings: 10th International Conference on Composite Science and Technology (ICCST/10), Lisbon, Portugal; 2-4 September 2015. Doi: 10.13140/RG.2.1.2784.2004 
[22] C. Le Gressus, F. Valin, M. Henriot, M. Gautier, J.P. Duraud, T.S. Sudarshan, R.G. Bommakanti, G. Blaise, Flashover in wide-band gap high purity insulators: Methodology and mechanisms, J. Appl. Phys. 69 (1991) 6325-6333.

[23] B. Vallayer, G. Blaise, D. Treheux, Space charge measurement in a dielectric material after irradiation with a $30 \mathrm{kV}$ electron beam: Application to singlecrystal oxide trapping properties, Rev. Sci. Instrum. 70 (1999) 3102-3112.

[24] B. Kchaou, C. Turki, M. Salvia, Z. Fakhfakh, D. Treheux, Role of fibre-matrix interface and fibre direction on dielectric behaviour of epoxy composites. Compos. Sci. Technol. 64 (2004) 1467-1475.

[25] C. Turki, B. Kechaou, D. Treheux, Z. Fakhfakh, M. Salvia, Fretting behaviour of unidirectional glass fibre-epoxy composites, influence of electric charge effects, Wear 257 (2004) 531-538.

[26] B. Kchaou, C. Turki, M. Salvia, Z. Fakhfakh, D. Treheux, Dielectric and friction behaviour of unidirectional glass fibre reinforced epoxy (GFRE), Wear 265 (2008) 763-771.

[27] B. Kechaou, M. Salvia, K. Benzarti, C. Turki, Z. Fakhfakh, D. Treheux, Role of fiber/matrix interphases on dielectric. friction, and mechanical properties of glass fiber-reinforced epoxy composites, J. Compos. Mater. 46 (2012) 131-144.

[28] B. Kchaou, M. Salvia, B. Beaugiraud, D. Juvé, Z. Fakhfakh, D. Treheux, Mechanical and dielectric characterization of hemp fibre reinforced polypropylene (HFRPP) by dry impregnation, Express Polym. Lett. 4 (2010) 171-182.

[29] A. Fazal, K.S. Fancey, Performance enhancement of nylon/Kevlar fiber composites through viscoelastically generated prestress, Polym. Compos. 35 (2014) 931-938.

[30] B. Wang, K.S. Fancey, Towards optimisation of load-time conditions for producing viscoelastically prestressed polymeric matrix composites, Compos. Part B 87 (2016) 336-342.

[31] S. Yotte, D. Breysse, J. Riss, S. Ghosh, Cluster characterisation in a metal matrix composite, Mater. Charact. 46 (2001) 211-219.

[32] B. Elsafi, Study of dynamic behavior of trapped charge in the insulating materials by using a new experimental approach, J. Electrostat. 72 (2014) 59-64.

[33] F.R. Jones, A review of interphase formation and design in fibre-reinforced composites, J. Adhesion Sci. Technol. 24 (2010) 171-202.

[34] G. Blaise, Charge localization and transport in disordered dielectric materials, J. Electrostat. 50 (2001) 69-89.

[35] G. Blaise, C. Le Gressus, Charging and flashover induced by surface polarization relaxation process, J. Appl. Phys. 69 (1991) 6334-6339.

[36] K.S. Fancey, A mechanical model for creep, recovery and stress relaxation in polymeric materials, J. Mater. Sci. 40 (2005) 4827-4831.

[37] H. Watanabe, Viscoelasticity and dynamics of entangled polymers, Prog. Polym. Sci. 24 (1999) 1253-1403. 


\section{Table 1}

Measured 1/d data (in $\mathrm{mm}^{-1}$ ) for resin-only (low and high air bubble concentrations) and composite samples; each value is shown as the mean \pm standard error from five samples.

\begin{tabular}{|c|c|c|c|c|c|c|}
\hline \multirow[b]{2}{*}{ Voltage (V) } & \multicolumn{2}{|c|}{$V_{\mathrm{f}}=0 \%$ (resin-only) } & \multicolumn{2}{|l|}{$V_{\mathrm{f}}=2 \%$} & \multicolumn{2}{|l|}{$V_{\mathrm{f}}=15 \%$} \\
\hline & Low $V_{\mathrm{b}}$ & High $V_{\mathrm{b}}$ & Test & Control & Test & Control \\
\hline 200 & $0.931 \pm 0.040$ & $0.292 \pm 0.025$ & $1.380 \pm 0.118$ & $0.968 \pm 0.006$ & $0.410 \pm 0.013$ & $0.411 \pm 0.007$ \\
\hline 300 & $1.317 \pm 0.061$ & $0.423 \pm 0.047$ & $2.003 \pm 0.129$ & $1.536 \pm 0.002$ & $1.050 \pm 0.065$ & $0.810 \pm 0.032$ \\
\hline 400 & $1.684 \pm 0.080$ & $0.575 \pm 0.062$ & $2.489 \pm 0.184$ & $2.015 \pm 0.003$ & $1.620 \pm 0.043$ & $1.075 \pm 0.072$ \\
\hline 500 & $2.012 \pm 0.094$ & $0.739 \pm 0.065$ & $2.851 \pm 0.206$ & $2.383 \pm 0.004$ & $2.180 \pm 0.047$ & $1.641 \pm 0.118$ \\
\hline 600 & $2.301 \pm 0.108$ & $0.892 \pm 0.069$ & $3.129 \pm 0.213$ & $2.686 \pm 0.006$ & $2.856 \pm 0.057$ & $2.096 \pm 0.178$ \\
\hline 700 & $2.552 \pm 0.118$ & $1.031 \pm 0.072$ & $3.364 \pm 0.246$ & $2.986 \pm 0.008$ & $3.466 \pm 0.065$ & $2.550 \pm 0.214$ \\
\hline 800 & $2.745 \pm 0.131$ & $1.170 \pm 0.078$ & $3.525 \pm 0.264$ & $3.130 \pm 0.008$ & $4.028 \pm 0.092$ & $2.951 \pm 0.234$ \\
\hline 900 & $2.889 \pm 0.138$ & $1.277 \pm 0.075$ & $3.611 \pm 0.255$ & $3.239 \pm 0.006$ & $4.357 \pm 0.166$ & $3.233 \pm 0.315$ \\
\hline 1000 & $2.957 \pm 0.133$ & $1.369 \pm 0.078$ & $3.658 \pm 0.264$ & $3.268 \pm 0.003$ & $4.408 \pm 0.152$ & $3.270 \pm 0.300$ \\
\hline
\end{tabular}

Table 2

Tensile test results from $2 \% V_{\mathrm{f}}$ and $15 \% V_{\mathrm{f}}$ samples showing peak stress and strain values at matrix fracture. SE is the standard error.

\begin{tabular}{|c|c|c|c|c|c|c|c|c|}
\hline \multirow[b]{2}{*}{ Sample No } & \multicolumn{2}{|c|}{$2 \% V_{\mathrm{f}}$ Peak stress $(\mathrm{MPa})$} & \multicolumn{2}{|c|}{$2 \% V_{\mathrm{f}}$ Strain at peak stress $(\%)$} & \multicolumn{2}{|c|}{$15 \% V_{\mathrm{f}}$ Peak stress $(\mathrm{MPa})$} & \multicolumn{2}{|c|}{$15 \% V_{\mathrm{f}}$ Strain at peak stress $(\%)$} \\
\hline & Test & Control & Test & Control & Test & Control & Test & Control \\
\hline 1 & 41.58 & 37.49 & 6.81 & 6.46 & 61.64 & 50.69 & 10.57 & 5.89 \\
\hline 2 & 44.08 & 43.61 & 6.74 & 6.42 & 51.44 & 60.24 & 6.21 & 11.86 \\
\hline 3 & 43.27 & 43.80 & 6.98 & 6.26 & 43.28 & 44.70 & 4.08 & 4.73 \\
\hline 4 & 43.14 & 44.37 & 6.83 & 6.48 & 70.76 & 53.60 & 14.18 & 8.35 \\
\hline 5 & 42.61 & 39.60 & 6.68 & 5.00 & 57.70 & 50.55 & 9.30 & 7.68 \\
\hline Increase $(\%)$ & \multicolumn{2}{|c|}{2.8} & \multicolumn{2}{|c|}{11.1} & \multicolumn{2}{|c|}{9.6} & \multicolumn{2}{|c|}{15.1} \\
\hline
\end{tabular}



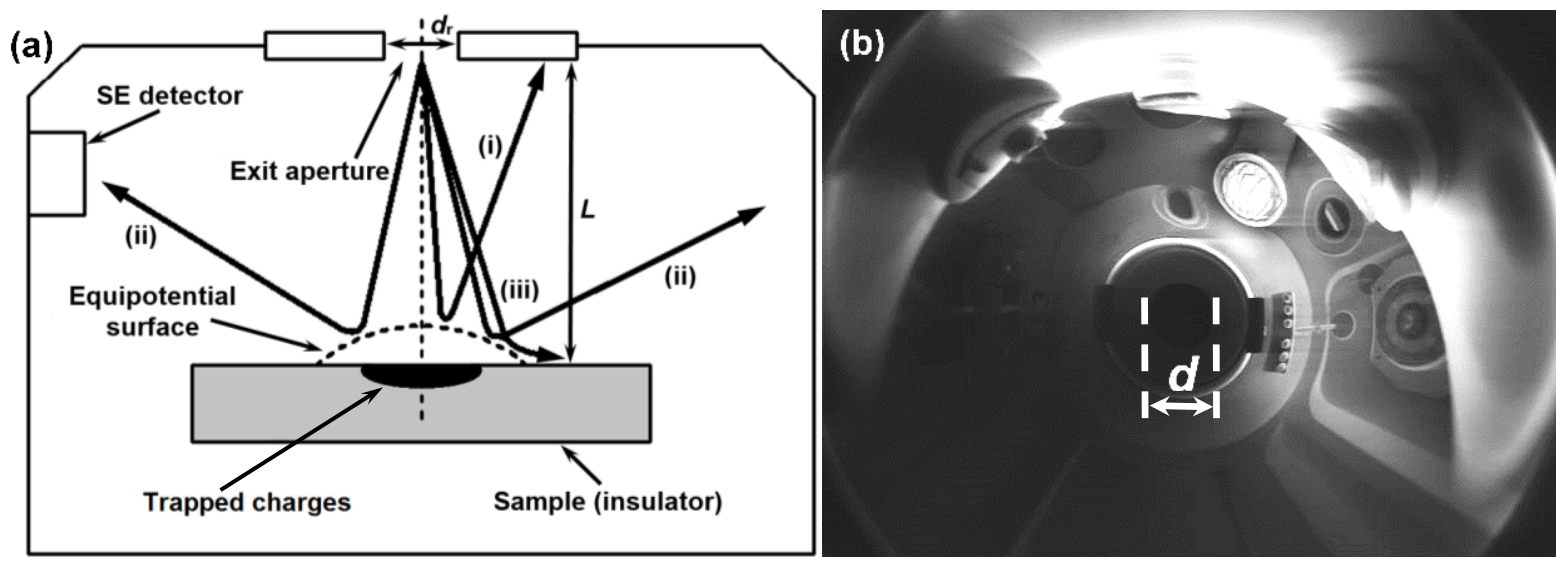

Fig. 1. The SEMME method. (a) Schematic of how trapped charges (following high voltage injection) within an insulating sample create an electric field, producing an electrostatic convex mirror; (b) Typical SEMME image taken from our chamber. In (a), electrons bounce off an equipotential surface, which may follow close to the direction of path (i) to give the central black disc in (b), or (ii) to give a mirror image of the chamber walls in (b), or (iii) to give a distorted image of the sample (re-drawn [24]). 


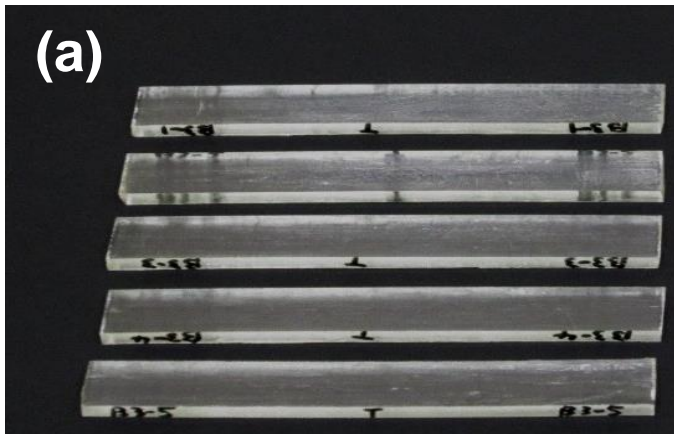

TEST

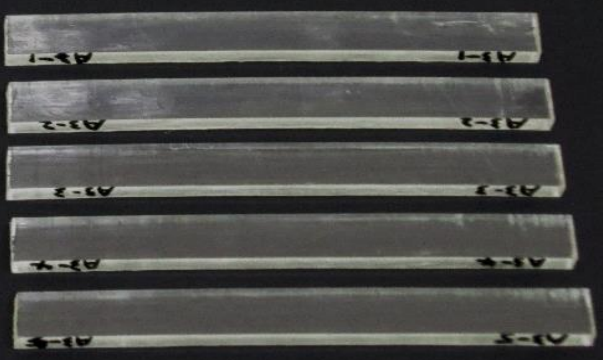

CONTROL

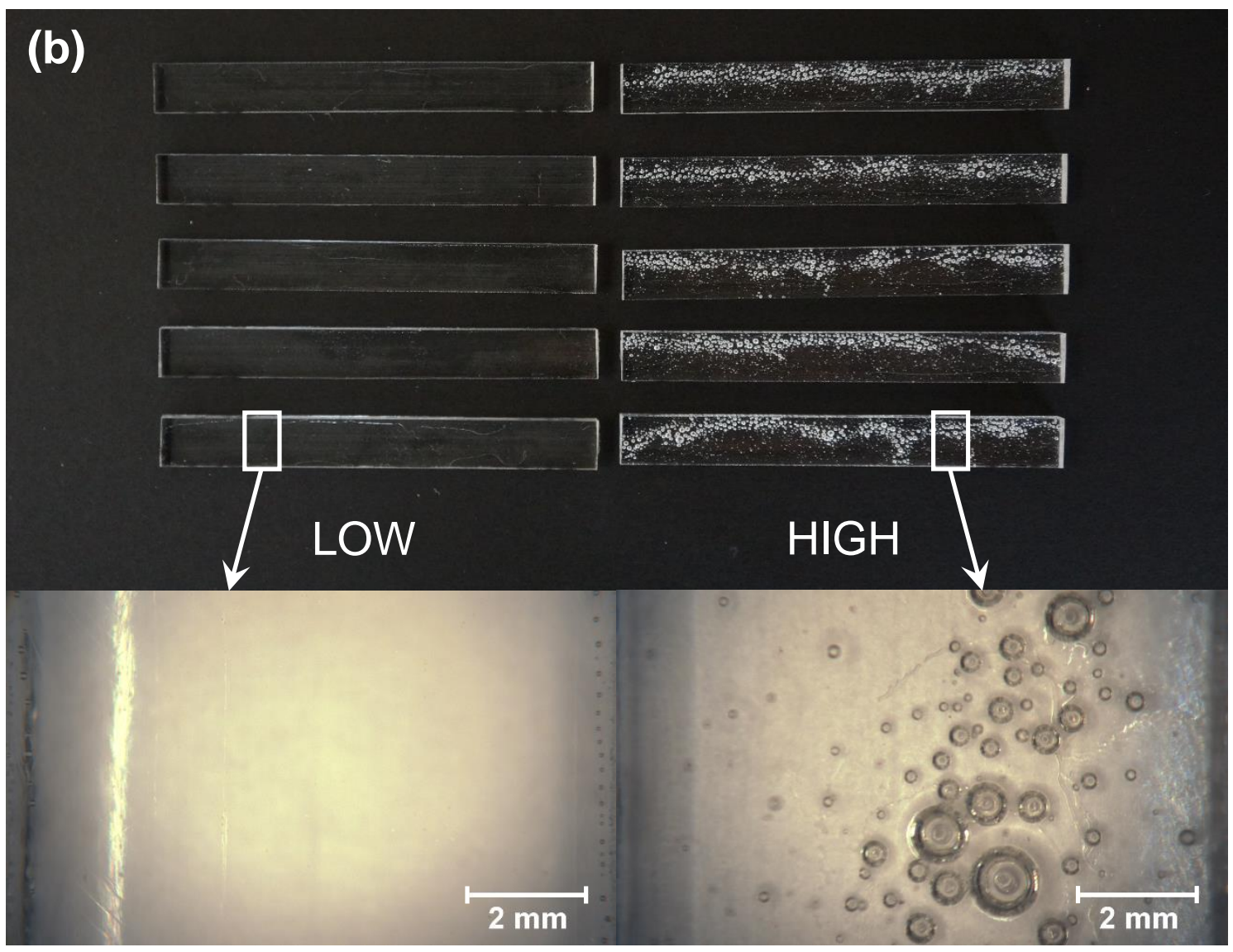

Fig. 2. Photographs of samples: (a) batch of test (prestressed) and control (nonprestressed) samples $\left(2 \% V_{\mathrm{f}}\right)$; (b) resin-only samples with low and high air bubble concentrations. 


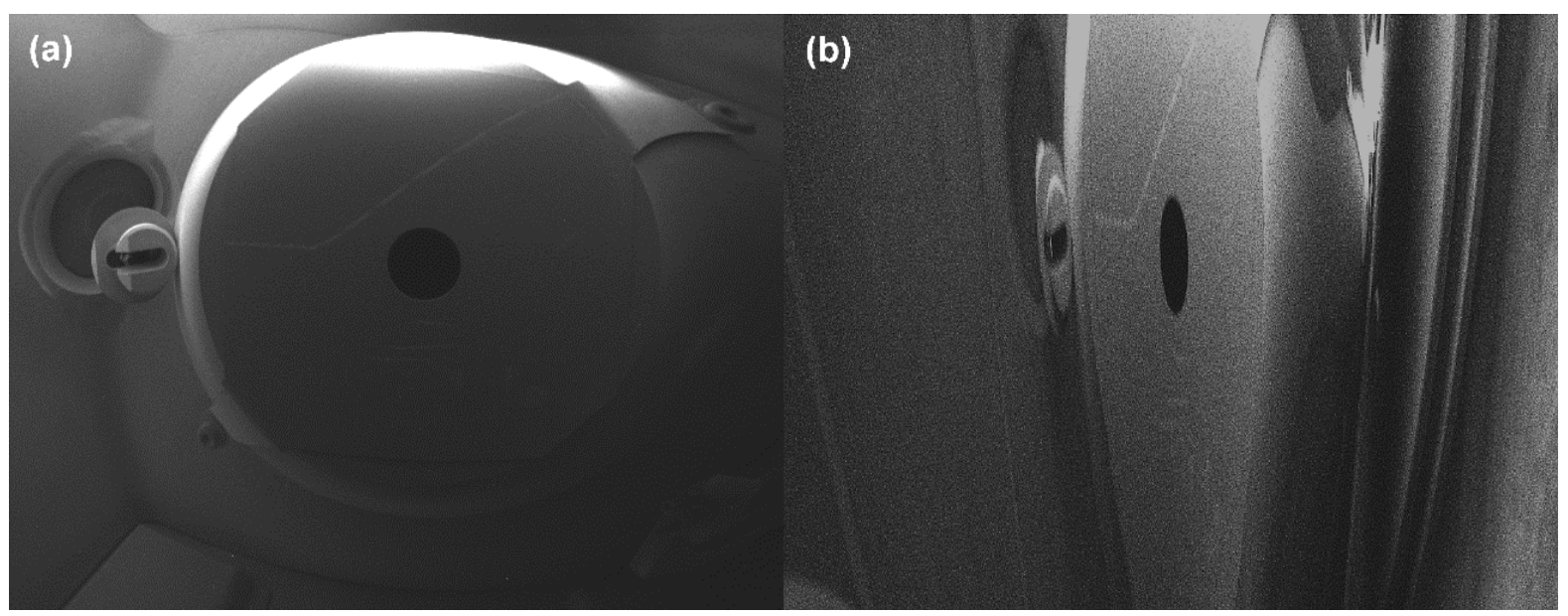

Fig. 3. Mirror images of the SEM gun exit aperture: (a) from a resin-only sample; (b) from a $15 \% V_{\mathrm{f}}$ sample. 
(a)
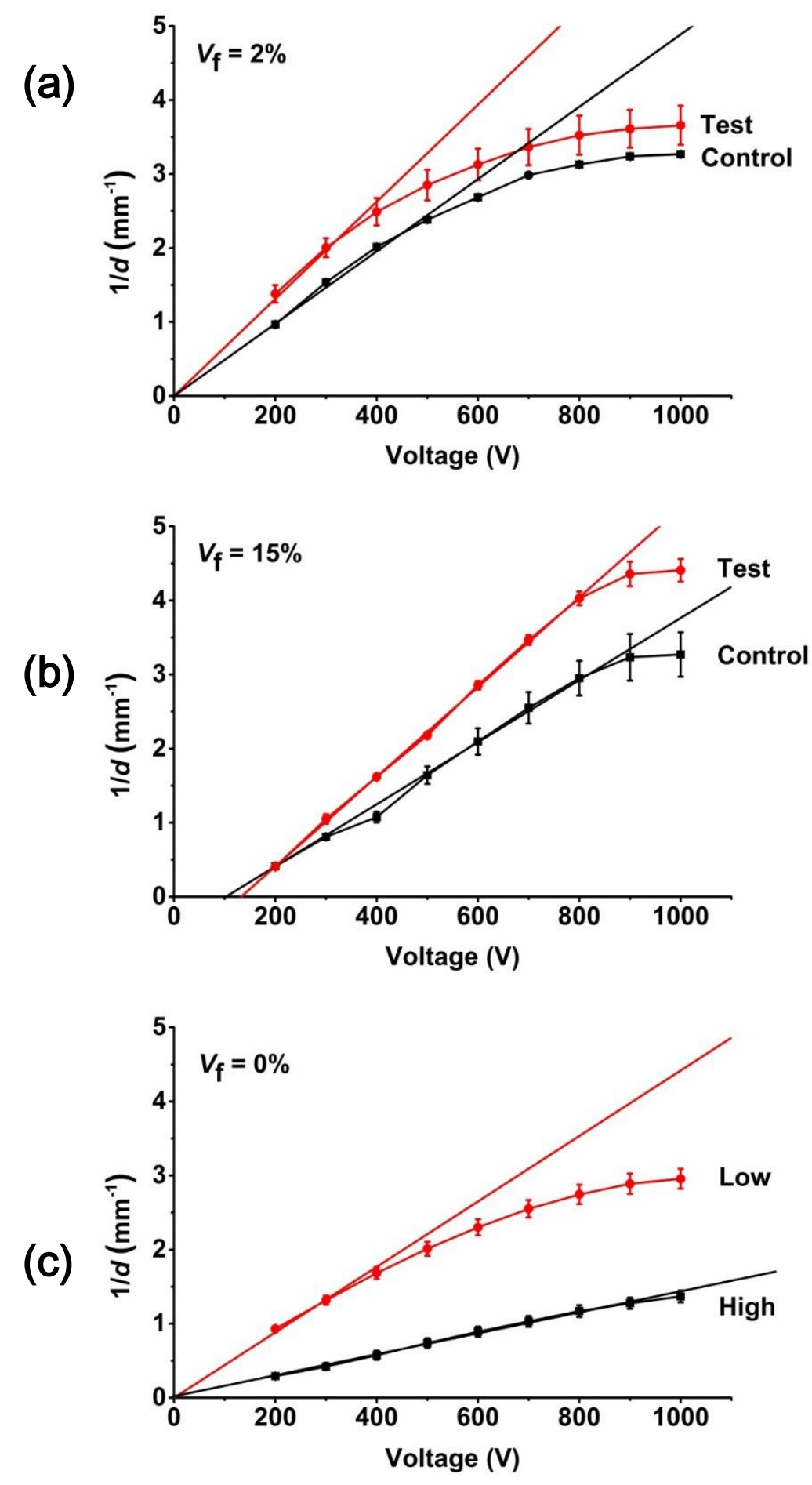

Fig. 4. SEMME plots of $1 / d$ versus accelerating voltage $V$ for (a) $2 \% V_{\mathrm{f}}$ samples, (b) $15 \% V_{\mathrm{f}}$ samples and (c) resin-only samples with low and high air bubble concentrations. Error bars represent standard error of the mean values ( 5 readings per data point); both linear and sub-linear regions can be observed. 

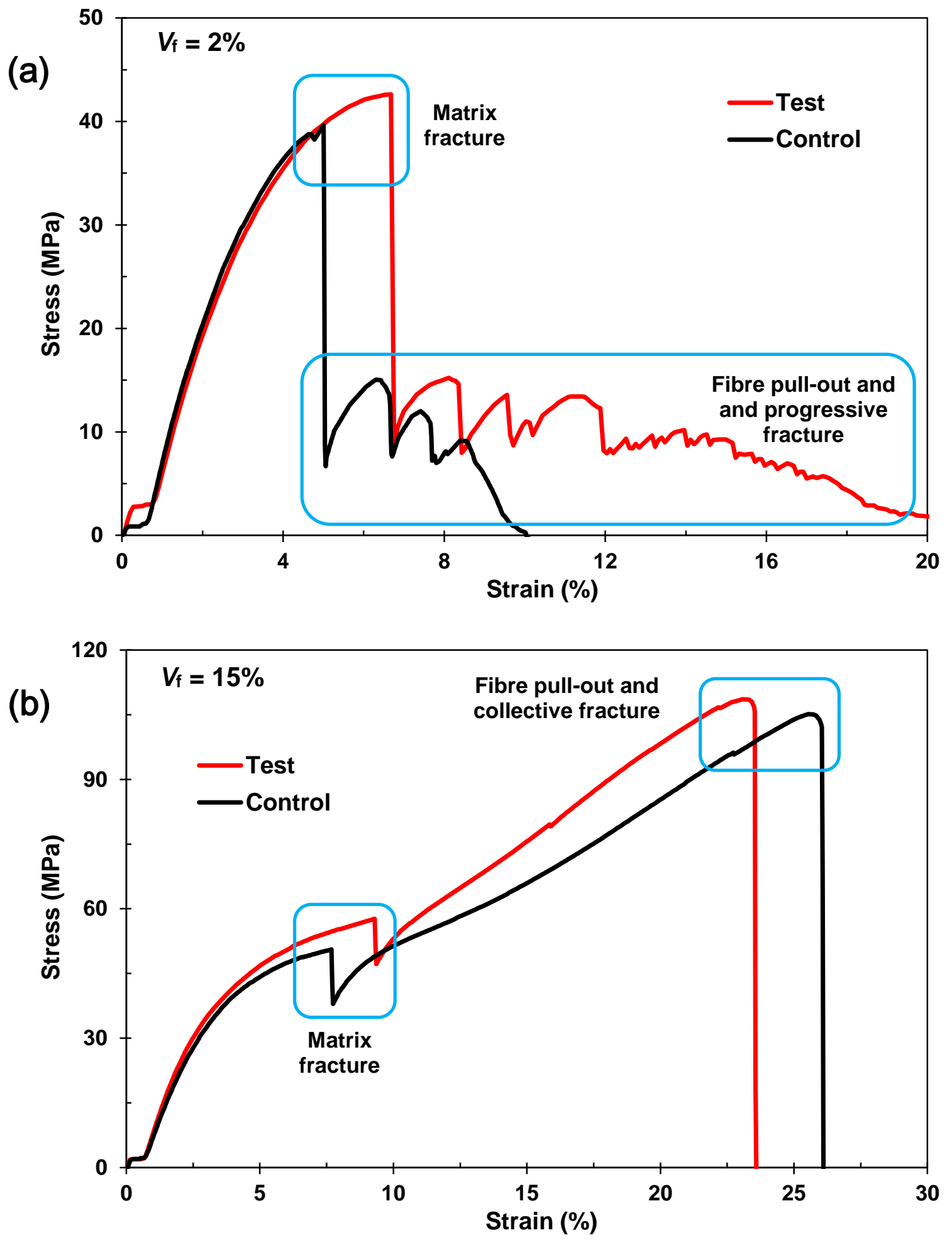

Fig. 5. Typical stress-strain curves from tensile tests on test and control samples at (a) $2 \% V_{\mathrm{f}}$ and (b) $15 \% V_{\mathrm{f}}$. 